\title{
On rainbow trees and cycles
}

\author{
Alan Frieze * $\quad$ Michael Krivelevich ${ }^{\dagger}$ \\ Submitted: Jan 4, 2007; Accepted: Apr 10, 2008; Published: Apr 18, 2008 \\ Mathematics Subject Classification: 05C15
}

\begin{abstract}
We derive sufficient conditions for the existence of rainbow cycles of all lengths in edge colourings of complete graphs. We also consider rainbow colorings of a certain class of trees.
\end{abstract}

\section{Introduction}

Let the edges of the complete graph $K_{n}$ be coloured so that no colour is used more than $\max \{b, 1\}$ times. We refer to this as a $b$-bounded colouring. We say that a subset $S$ of the edges of $K_{n}$ is rainbow coloured if each edge of $S$ is of a different colour. Various authors have considered the question of how large can $b=b(n)$ be so that any $b$-bounded edge colouring contains a rainbow Hamilton cycle. It was shown by Albert, Frieze and Reed [1] (see Rue [7] for a correction in the claimed constant) that $b$ can be as large as $n / 64$. This confirmed a conjecture of Hahn and Thomassen [5]. Our first theorem discusses the existence of rainbow cycles of all sizes. We give a kind of a pancyclic rainbow result.

Theorem 1 There exists an absolute constant $c>0$ such that if an edge colouring of $K_{n}$ is cn-bounded then there exist rainbow cycles of all sizes $3 \leq k \leq n$.

Having dealt with cycles, we turn our attention to trees.

Theorem 2 Given a real constant $\varepsilon>0$ and a positive integer $\Delta$, there exists a constant $c=c(\varepsilon, \Delta)$ such that if an edge colouring of $K_{n}$ is cn-bounded, then it contains a rainbow copy of every tree $T$ with at most $(1-\varepsilon) n$ vertices and maximum degree $\Delta$.

*Department of Mathematical Sciences, Carnegie Mellon University, Pittsburgh PA15213, U.S.A. Supported in part by NSF grant CCF0502793.

${ }^{\dagger}$ School of Mathematical Sciences, Raymond and Beverly Sackler Faculty of Exact Sciences, Tel Aviv University, Tel Aviv 69978, Israel. E-mail: krivelev@post.tau.ac.il. Research supported in part by USAIsrael BSF Grants 2002-133 and 2006-322, by grant 526/05 from the Israel Science Foundation and by the Pazy memorial award. 
We conjecture that that there is a constant $c=c(\Delta)$ such that every $c n$-bounded edge colouring of $K_{n}$ contains a rainbow copy of every spanning tree of $K_{n}$ which has maximum degree at most $\Delta$. We are far from proving this and give a small generalisation of the known case where the tree in question is a Hamilton path. Let $T^{*}$ be an arbitrary rooted tree with $\nu_{0}$ nodes. Assume that $\nu_{0}$ divides $n$ and let $\nu_{1}=n / \nu_{0}$. We define $T\left(\nu_{1}\right)$ as follows: It has a spine which is a path $P=\left(x_{0}, x_{1}, \ldots, x_{\nu_{1}-1}\right)$ of length $\nu_{1}-1$. We then have $\nu_{1}$ vertex disjoint copies $T_{0}, T_{1}, \ldots, T_{\nu_{1}-1}$ of $T^{*}$, where $T_{i}$ is rooted at $x_{i}$ for $i=0,1, \ldots, \nu_{1}-1$. $T(\nu)$ has $n$ vertices. The edges of $T\left(\nu_{1}\right)$ are of two types, spine-edges in $P$ and teeth-edges.

We state our theorem as

Theorem 3 If an edge colouring of $K_{n}$ is $k$-bounded and $\left(\begin{array}{c}\nu_{1}-2 \\ 2\end{array}\right)>16 k n$ then there exists a rainbow copy of every possible $T\left(\nu_{1}\right)$.

\section{Proof of Theorem 1}

We will not attempt to maximise $c$ as we will be far from the optimum.

The following lemma is enough to prove the theorem:

\section{Lemma 4}

(a) Let $c_{0}=2^{-7}$ and suppose that $n \geq 2^{21}$. Then every $2 c_{0} n$-bounded edge colouring of $K_{n}$ contains rainbow cycles of length $k, n / 2 \leq k \leq n$.

(b) If $n \geq e^{1000}$ and $c n \geq n^{2 / 3}$ and an edge colouring of $K_{n}$ is cn-bounded, then there exists a set $S \subseteq[n]$ such that $|S|=N=n / 2$ and the induced colouring of the edges of $S$ is $c^{\prime} N$-bounded where $c^{\prime}=c\left(1+1 /(\ln n)^{2}\right)$.

We will first show that the lemma implies the theorem. Assume first that $n \geq e^{1000}$. We let $N_{i}=2^{-i} n$ for $0 \leq i \leq r=\left\lfloor\log _{2}\left(n e^{-1000}\right)\right\rfloor$ and note that $N_{i} \geq e^{1000}>2^{21}$ for all $i \leq r$. Now define a sequence $c_{0}, c_{1}, c_{2}, \ldots, c_{r}$ by

$$
c_{i+1}=c_{i}\left(1+\frac{1}{\left(\ln N_{i}\right)^{2}}\right) .
$$

Then for $i \geq 1$ we have:

$$
\begin{aligned}
c_{i} & =c_{0} \prod_{s=1}^{i}\left(1+\frac{1}{(\ln n-s \ln 2)^{2}}\right) \\
& \leq c_{0} \exp \left\{\frac{1}{(\ln n)^{2}} \sum_{s=1}^{i} \frac{1}{\left(1-\frac{s}{\log _{2} n}\right)^{2}}\right\}
\end{aligned}
$$




$$
=c_{0} \exp \left\{\left(\frac{\log _{2} n}{\ln n}\right)^{2} \sum_{s=1}^{i} \frac{1}{\left(\log _{2} n-s\right)^{2}}\right\} .
$$

Then for all $0 \leq i \leq r$ we have:

$$
c_{0} \leq c_{i} \leq c_{0} \exp \left\{\left(\frac{\log _{2} n}{\ln n}\right)^{2} \sum_{t=21}^{\infty} \frac{1}{t^{2}}\right\} \leq c_{0} \exp \left\{2.1 \int_{t=20}^{\infty} t^{-2} d t\right\}=c_{0} \exp \left\{\frac{2.1}{20}\right\} \leq 2 c_{0} .
$$

Furthermore, for $0 \leq i \leq r$ we have $n / 2^{r}>2^{21}$ and so

$$
c_{i} N_{i}^{1 / 3} \geq \frac{c_{0} n^{1 / 3}}{2^{i / 3}} \geq 1
$$

which implies that $c_{i} N_{i} \geq N_{i}^{2 / 3}$.

Assume now we are given a $c_{0} n$-bounded coloring of $K_{n}$ and that $n \geq e^{1000}$. Then by part (a) of the lemma we can find rainbow cycles of length $k, n / 2 \leq k \leq n$. By part (b) there exists a subset $S,|S|=n / 2=N$, such that the induced coloring on $S$ is $c_{1} n$-bounded. Now we can apply part (a) of the lemma to the induced subgraph $G[S]$ to find rainbow cycles of length $k, n / 4 \leq k \leq n / 2$. We can continue this halving process for $r$ steps, thus finding rainbow cycles of length $k, N_{r} \leq k \leq n$ where $e^{1000} \leq N_{r} \leq 2 e^{1000}$.

To summarise: Assuming the truth of Lemma 4, if $n \geq e^{1000}$ and $c \leq 2^{-7}$ then any cn-bounded coloring of $K_{n}$ contains a rainbow cycle of length $2 e^{1000} \leq k \leq n$.

Up to this point, the value of $c$ is quite reasonable. We now choose a very small value of $c$ in order to finish the proof without too much more effort.

Suppose now that $c \leq e^{-3001}, n \geq e^{1000}$ and $3 \leq k \leq \min \left\{2 e^{1000}, n\right\}$. Suppose that $K_{n}$ is edge colored with $q$ colors and that color $i$ is used $m_{i} \leq c n$ times. Choose a set $S$ of $k$ vertices. Let $\mathcal{E}$ be the event $S$ contains two edges of the same color. at random. Then,

$$
\begin{aligned}
\operatorname{Pr}(\mathcal{E}) & \leq\left(\begin{array}{c}
k \\
2
\end{array}\right)^{2} \sum_{i=1}^{q}\left(\frac{m_{i}}{\left(\begin{array}{c}
n \\
2
\end{array}\right)}\right)^{2}+\left(\begin{array}{l}
k \\
3
\end{array}\right) \sum_{i=1}^{q} \frac{\left(\begin{array}{c}
m_{i} \\
2
\end{array}\right)}{\left(\begin{array}{c}
n \\
3
\end{array}\right)} \\
& \leq\left(\begin{array}{c}
k \\
2
\end{array}\right)^{2} \frac{\left(\begin{array}{c}
n \\
2
\end{array}\right)}{c n}\left(\frac{c n}{\left(\begin{array}{c}
n \\
2
\end{array}\right)}\right)^{2}+\left(\begin{array}{l}
k \\
3
\end{array}\right) \frac{\left(\begin{array}{c}
n \\
2
\end{array}\right)}{c n} \frac{\left(\begin{array}{c}
c n \\
2
\end{array}\right)}{\left(\begin{array}{c}
n \\
3
\end{array}\right)} \\
& \leq \frac{c k^{2}}{n-1}+\frac{c k^{3}}{4} \\
& <1 .
\end{aligned}
$$

The two sums in (1) correspond to having two disjoint edges with the same color and to two edges of the same color sharing a vertex, respectively.

All that is left is the case $n \leq e^{1000}$ but now $c$ is so small that $c n<1$ and all edges have distinct colors. 


\subsection{Proof of Lemma 4}

Part (a) follows immediately from [1] $\left(n \geq 2^{21}\right.$ is easily large enough for the result there to hold). We can apply the main theorem of that paper to any subset of $[n]$ with at least $n / 2$ vertices.

We now prove part (b). Let $S$ be a random $n / 2$-subset of $[n]$. Now for each colour $i$ we orient the $i$-coloured edges of $K_{n}$ so that for each $v \in[n]$,

$$
\left|d_{i}^{+}(v)-d_{i}^{-}(v)\right| \leq 1
$$

where $d_{i}^{+}(v)$ (resp. $\left.d_{i}^{-}(v)\right)$ is the out-degree (resp. in-degree) of $v$ in the digraph $D_{i}=$ $\left([n], E_{i}\right)$ induced by the edges of colour $i$. Now fix a colour $i$ and let

$$
L_{i}=\left\{v: d_{i}^{+}(v) \geq(\ln n)^{6}\right\}
$$

Then with $(v, w)$ denoting an edge oriented from $v$ to $w$ we let

$$
\begin{aligned}
& A_{1}=\left\{(v, w) \in E_{i}: v \in L_{i}\right\} \\
& A_{2}=\left\{(v, w) \in E_{i}: v \notin L_{i}, w \in L_{i} \text { and } \exists \geq(\ln n)^{6} \text { edges of colour } i \text { from } \bar{L}_{i} \text { to } w\right\} \\
& A_{3}=E_{i} \backslash\left(A_{1} \cup A_{2}\right) .
\end{aligned}
$$

Let $\left|A_{j}\right|=\alpha_{j} n$ where $\alpha_{1}+\alpha_{2}+\alpha_{3} \leq c$.

Let $Z_{j}, j=1,2,3$, be the number of edges of $A_{j}$ which are entirely contained in $S$ and let $Z=Z_{1}+Z_{2}+Z_{3}$. We write

$$
Z_{1}=\sum_{v \in L_{i}} 1_{v \in S} X_{1, v}
$$

where $X_{1, v}$ is the number of neighbours of $v$ in $D_{i}$ that are included in $S$.

Now

$$
\operatorname{Pr}\left(X_{1, v} \geq \frac{1}{2} d_{i}^{+}(v)+\frac{1}{4} d_{i}^{+}(v)^{1 / 2} \ln n\right) \leq e^{-(\ln n)^{2} / 24} .
$$

This follows from the Chernoff bounds (more precisely, using Hoeffding's lemma [6] about sampling without replacement).

Note that

$$
\frac{1}{2} d_{i}^{+}(v)+\frac{1}{4} d_{i}^{+}(v)^{1 / 2} \ln n \leq \frac{1}{2} d_{i}^{+}(v)\left(1+\frac{1}{2(\ln n)^{2}}\right) .
$$

So, on using $n \geq e^{1000}$, we see that with probability at least

$$
1-n e^{-(\ln n)^{2} / 24}=1-n^{1-(\ln n) / 24} \geq 9 / 10
$$

we have

$$
Z_{1} \leq \frac{1}{2} \alpha_{1} n\left(1+\frac{1}{2(\ln n)^{2}}\right)
$$


The edges of $A_{2}$ are dealt with in exactly the same manner and we have that with probability at least $9 / 10$,

$$
Z_{2} \leq \frac{1}{2} \alpha_{2} n\left(1+\frac{1}{2(\ln n)^{2}}\right)
$$

To deal with $Z_{3}$ we observe that if we delete a vertex $v$ of $S$ then $Z_{3}$ can change by at most $2(\ln n)^{6}$. This is because the digraph induced by $A_{3}$ has maximum in-degree and out-degree bounded by $(\ln n)^{6}$. Applying a version of Azuma's inequality that deals with sampling without replacement (see for example Lemma 11 of [4]) we see that for $t>0$,

$$
\operatorname{Pr}\left(Z_{3} \geq \frac{1}{4} \alpha_{3} n+t\right) \leq \exp \left\{-\frac{2 t^{2}}{n(\ln n)^{12}}\right\} .
$$

So, putting $t=n^{3 / 5}$ and using $n \geq e^{1000}$ and $c n \geq n^{2 / 3}$ we see that with probability at least $9 / 10$,

$$
Z \leq \frac{1}{2}\left(\alpha_{1}+\alpha_{2}\right) n\left(1+\frac{1}{2(\ln n)^{2}}\right)+\frac{1}{4} \alpha_{3} n+n^{3 / 5} \leq \frac{1}{2} c n\left(1+\frac{1}{(\ln n)^{2}}\right) .
$$

So, with probability at least $7 / 10$ the colouring of the edges of $S$ is $c\left(1+1 /(\ln n)^{2}\right) n / 2$ bounded and Lemma 4 is proved.

\section{Proof of Theorem 2}

We proceed as follows. We choose a large $d=d(\varepsilon, \Delta)>0$ and a small $c \ll 1 / d^{3 / 2}$ and consider a $c n$-bounded edge colouring of $K_{n}$. We then define $G_{1}=G_{n, p}, p=d / n$. We remove any edge of $G_{1}$ which has the same colour as another edge of $G_{1}$. Call the remaining graph $G_{2}$. The edge set of $G_{2}$ is rainbow coloured. We then remove vertices of low and high degree to obtain a graph $G_{3}$. We then show that whp $G_{3}$ satisfies the conditions of a theorem of Alon, Krivelevich and Sudakov [2], implying that $G_{3}$ contains a copy of every tree with $\leq(1-\varepsilon) n$ vertices and maximum degree $\leq \Delta$. The theorem we need from [2] is the following:

Definition: Given two positive numbers $a_{1}$ and $a_{2}<1$, a graph $G=(V, E)$ is called an $\left(a_{1}, a_{2}\right)$-expander if every subset of vertices $X \subseteq V$ of size $|X| \leq a_{1}|V|$ satisfies $\left|N_{G}(X)\right| \geq$ $a_{2}|X|$. Here $N_{G}(X)$ is the set of vertices in $V(G) \backslash X$ that are neighbours of vertices in $X$.

Theorem 5 Let $\Delta \geq 2,0<\varepsilon<1 / 2$. Let $H$ be a graph on $N$ vertices of minimum degree $\delta_{H}$ and maximum degree $\Delta_{H}$. Suppose that

T1

$$
N \geq \frac{480 \Delta^{3} \ln (2 / \varepsilon)}{\varepsilon}
$$


T2

$$
\Delta_{H}^{2} \leq \frac{1}{K} e^{\delta_{H} /(8 K)-1} \text { where } K=\frac{20 \Delta^{2} \ln (2 / \varepsilon)}{\varepsilon}
$$

T3 Every subgraph $H_{0}$ of $H$ with minimum degree at least $\frac{\varepsilon \delta_{H}}{40 \Delta^{2} \ln (2 / \varepsilon)}$ is a $\left(\frac{1}{2 \Delta+2}, \Delta+1\right)$ expander.

Then $H$ contains a copy of every tree with $\leq(1-\varepsilon) N$ vertices and maximum degree $\leq \Delta$.

We now get down to details. In the following we assume that $c d \ll 1 \ll d$. We will prove that whp,

P1 The number of edges using repeated colours is at most $d^{2} c n$.

P2 Every set $X \subseteq[n],|X| \leq n / d^{1 / 5}$ contains less than $\alpha d|X|$ edges of $G_{1}$ where, with $\Delta=2 d$,

$$
\alpha=\frac{\varepsilon}{\left(100 \Delta^{2}(\Delta+2) \ln (2 / \varepsilon)\right)} .
$$

P3 $G_{1}$ contains at most $n e^{-d / 10}$ vertices of degree outside $[d / 2,2 d]$.

P4 Every pair of disjoint sets $S, T \subseteq[n]$ of size $n / d^{1 / 4}$ are joined by at least $d^{1 / 2} n / 2$ edges in $G_{1}$.

Before proving that P1-P4 hold whp, let us show that they are sufficient for our purposes. Starting with $G_{1}=G_{n, p}$ we remove all edges using repeated colours to obtain $G_{2}$. Then let $X_{0}$ denote the set of vertices of $G_{2}$ whose degree is not in $[d / 3,2 d]$. It follows from P1,P3 that

$$
\left|X_{0}\right| \leq n\left(e^{-d / 10}+12 c d\right)
$$

Note that $12 c d n$ bounds the number of vertices that lose more than $d / 6$ edges in going from $G_{1}$ to $G_{2}$.

Now consider a sequence of sets $X_{0}, X_{1}, \ldots$, where $X_{i}=X_{i-1} \cup\left\{x_{i}\right\}$ and $x_{i}$ has at least $2 \alpha d$ neighbours in $X_{i-1}$. We continue this process as long as possible. Let $G_{3}$ be the resulting graph. We claim that the process stops before $i$ reaches $\left|X_{0}\right|$. If not, we have a set with $2\left|X_{0}\right|$ vertices and at least $2 \alpha d\left|X_{0}\right|$ edges. For this we need $2\left|X_{0}\right| \geq n / d^{1 / 5}$ (see P2) and this contradicts (2) if $d$ is large and $c<1 / d^{2}$.

Thus $H=G_{3}$ has at least $n\left(1-2\left(e^{-d / 10}+12 c d\right)\right)$ vertices and this implies that $\mathbf{T} \mathbf{1}$ holds. Also,

$$
d(1 / 3-2 \alpha) \leq \delta_{H} \leq \Delta_{H} \leq 2 d .
$$

So if $d \gg K^{2}$, T2 will also hold.

Now consider a subgraph $\Gamma$ of $H$ which has minimum degree at least $\beta d$ where $\beta=$ $2(\Delta+2) \alpha$. Let $\nu=|V(\Gamma)|$. Choose $S \subseteq V(\Gamma)$ where $|S| \leq \frac{\nu}{2 \Delta+2}$ and let $T=N_{\Gamma}(S)$. Suppose also that $|T|<(|\Delta|+1)|S|$. 
Suppose first that $|S| \geq n / d^{1 / 4}$. Then $|S \cup T| \leq \nu(\Delta+2) /(2 \Delta+2)$ and so $Y=V(\Gamma) \backslash(S \cup T)$ satisfies $|Y| \geq|S| \geq n / d^{1 / 4}$. The fact that there are no $S: Y$ edges contradicts $\mathbf{P} \mathbf{1}, \mathbf{P} 4$.

Now assume that $1 \leq|S| \leq n / d^{1 / 4}$. Then $|S \cup T| \leq(\Delta+2) n / d^{1 / 4} \leq n / d^{1 / 5}$ and $S \cup T$ contains at least $\beta d|S| / 2 \geq \alpha d|S \cup T|$ edges, contradicting P2.

Thus, $\Gamma$ is $\left(\frac{1}{2 \Delta+2}, \Delta+1\right)$-expander and the minimum degree requirement is $\beta d$ which is weaker than required by $\mathbf{T} 3$.

It only remains to verify $\mathbf{P} \mathbf{1}-\mathbf{P} \mathbf{4}$ :

P1: Let $Z$ denote the number of edges using repeated colours. Let there be $m_{i} \leq c n$ edges with colour $i$ for $i=1,2, \ldots, \ell$. Then

$$
\mathbf{E}(Z) \leq \sum_{i=1}^{\ell}\left(\begin{array}{c}
m_{i} \\
2
\end{array}\right) p^{2} \leq \frac{\left(\begin{array}{c}
n \\
2
\end{array}\right)}{c n}\left(\begin{array}{c}
c n \\
2
\end{array}\right) \frac{d^{2}}{n^{2}} \leq \frac{c d^{2}}{4} n .
$$

Now whp $G_{1}$ has at most $d n$ edges and changing one edge can only change $Z$ by at most 2. So, by Azuma's inequality, we have

$$
\operatorname{Pr}(Z \geq \mathbf{E}(Z)+t) \leq \exp \left\{-\frac{2 t^{2}}{4 d n}\right\}
$$

and we get (something stronger than) $\mathbf{P} 1$ by taking $t=n^{3 / 4}$.

P2: The probability $\mathbf{P 2}$ fails is at most

$$
\left.\sum_{k=2 \alpha d}^{n / d^{1 / 5}}\left(\begin{array}{l}
n \\
k
\end{array}\right)\left(\begin{array}{c}
k \\
2
\end{array}\right)\right) p^{\alpha d k} \leq \sum_{k=2 \alpha d}^{n / d^{1 / 5}}\left(\left(\frac{k}{2 n}\right)^{\alpha d-1}\left(\frac{e}{\alpha}\right)^{\alpha d} e\right)^{k}=o(1) .
$$

P3: If now $Z$ is the number of vertices with degrees outside $[d / 2,2 d]$ then the Chernoff bounds imply that

$$
\mathbf{E}(Z) \leq n\left(e^{-d / 8}+e^{-d / 3}\right),
$$

and Azuma's inequality will complete the proof.

$\mathbf{P}$ 4: The probability $\mathbf{P} \mathbf{4}$ fails is at most

$$
\left(\begin{array}{c}
n \\
n / d^{1 / 4}
\end{array}\right)^{2} \sum_{k=0}^{d^{1 / 2} n / 2}\left(\begin{array}{c}
n^{2} / d^{1 / 2} \\
k
\end{array}\right) p^{k}(1-p)^{n^{2} / d^{1 / 2}-k} \leq 4^{n} e^{-d^{1 / 2} n / 8}=o(1) .
$$

\section{Proof of Theorem 3}

We will use the lop-sided Lovász local lemma as in Erdős and Spencer [3] and in Albert, Frieze and Reed [1]. We state the lemma as 
Lemma 6 Let $A_{1}, A_{2}, \ldots, A_{N}$ denote events in some probability space. Suppose that for each $i$ there is a partition of $[N] \backslash\{i\}$ into $X_{i}$ and $Y_{i}$. Let $m=\max \left\{\left|Y_{i}\right|: i \in[N]\right\}$ and $\beta=\max \left\{\operatorname{Pr}\left(A_{i} \mid \bigcap_{j \in S} \bar{A}_{j}\right): i \in[N], S \subseteq X_{i}\right\}$. If $4 m \beta<1$ then $\operatorname{Pr}\left(\bigcap_{i=1}^{n} \bar{A}_{i}\right)>0$.

Suppose now that we have a $k$-bounded colouring of $K_{n}$ and that $H$ is chosen uniformly from the set of all copies of $T(\nu)$ in $K_{n}$ where $T$ is an arbitrary rooted tree with $\nu$ vertices. We show that the probability that $H$ is a rainbow copy is strictly positive.

Let $\left\{e_{i}, f_{i}\right\}, i=1,2, \ldots, N$, be an enumeration of all pairs of edges of $K_{n}$ where $e_{i}, f_{i}$ have the same colour (thus $N=\sum_{\ell}\left(\begin{array}{c}n_{\ell} \\ 2\end{array}\right)$ where $n_{\ell}$ is the number of edges of colour $\ell$ ). Let $A_{i}$ be the event $H \supset\left\{e_{i}, f_{i}\right\}$ for $i=1,2, \ldots, N$. We apply Lemma 6 with the definition

$$
Y_{i}=\left\{j \neq i:\left(e_{j} \cup f_{j}\right) \cap\left(e_{i} \cup f_{i}\right) \neq \emptyset\right\} .
$$

With this definition

$$
m \leq 4 k n \text {. }
$$

We estimate $\beta$ as follows: Fix $i, S \subseteq X_{i}$. We show that for each $T \in \mathcal{T}_{1}=A_{i} \cap \bigcap_{j \in S} \bar{A}_{j}$ (this means that $T$ is a copy of $T\left(\nu_{0}, \nu_{1}\right)$ containing both $e_{i}, f_{i}$ and at most one edge from each pair $e_{j}, f_{j}$ for $j \in S$ ) there exists a set $S(T) \subseteq \mathcal{T}_{2}=\bar{A}_{i} \cap \bigcap_{j \in S} \bar{A}_{j}$ such that (i) $|S(T)|>4 k n$ and (ii) $S(T) \cap S\left(T^{\prime}\right)=\emptyset$ for $T \neq T^{\prime} \in \mathcal{T}_{1}$. This shows that

$$
\operatorname{Pr}\left(A_{i} \mid \bigcap_{j \in S} \bar{A}_{j}\right) \leq \frac{1}{4 m+1}
$$

and proves the theorem.

Fix $H \in \mathcal{T}_{1}$. If $e=\left(x_{i}, x_{i+1}\right)$ and $f=\left(x_{j}, x_{j+1}\right)$ are both spine-edges where $j-i \geq 2$, we define the tree $F_{\text {spine }}(H ; e, f)$, which is also a copy of $T(\nu)$, as follows: We delete $e, f$ from $H$ and replace them by $\left(x_{i}, x_{j}\right)$ and $\left(x_{i+1}, x_{j+1}\right)$. Suppose now that $e=(a, b) \in T_{i} \backslash x_{i}$ and $f=(c, d) \in T_{j} \backslash x_{j}$ are both teeth-edges and that $\phi(e)=f$ in some isomorphism from $T_{i}$ to $T_{j}$. Then we define $F_{\text {teeth }}(H ; e, f)$ as follows: We delete $e, f$ from $H$ and replace them by $(a, d)$ and $(b, c)$ to get another copy of $T(\nu)$.

Observe that if $f \neq f_{i}$ then $H^{\prime}=F_{\sigma}\left(H ; e_{i}, f\right) \in \mathcal{T}_{2}$ for $\sigma \in\{$ spine, teeth $\}$. This is because $e_{i}$ is not an edge of $H^{\prime}$ and the edges that we added are all incident with $e_{i}$. We cannot therefore have caused the occurrence of $A_{j}$ for any $j \in X_{i}$. Similarly, $F_{\sigma}\left(H^{\prime} ; f_{i}, g\right) \in \mathcal{T}_{2}$ for $g \neq e_{i}$.

We use $F_{\text {spine }}, F_{\text {teeth }}$ to construct $S(H)$ as follows: We choose an edge $f \neq f_{i}$ of the same type as $e_{i}$ and construct $H^{\prime}=F_{\sigma}\left(H ; e_{i}, f\right)$ for the relevant $\sigma$. We then choose $g \neq e_{i}$ of the same type as $f_{i}$ and construct $H^{\prime \prime}=F_{\sigma^{\prime}}\left(H^{\prime} ; f_{i}, g\right)$. In this way we construct $S(H) \subseteq \mathcal{T}_{2}$ containing at least $\left(\begin{array}{c}\nu_{1}-2 \\ 2\end{array}\right)$ distinct copies of $T\left(\nu_{1}\right)$.

Notice that knowing $e_{i}, f_{i}$ allows us to construct $H^{\prime}$ from $H^{\prime \prime}$ and then $H$ from $H^{\prime}$. This shows that $S(H) \cap S\left(H^{\prime}\right)=\emptyset$. After this, all we have to do is choose $k, \nu_{1}$ so that $\left(\begin{array}{c}\nu_{1}-2 \\ 2\end{array}\right)>16 k n$ in order to finish the proof of Theorem 3. 


\section{References}

[1] M. Albert, A.M. Frieze and B. Reed, Multicoloured Hamilton Cycles, Electronic Journal of Combinatorics 2 (1995), publication R10.

[2] N. Alon, M. Krivelevich and B. Sudakov, Embedding nearly-spanning bounded degree trees, Combinatorica, to appear.

[3] P. Erdős and J. Spencer, Lopsided Lovász Local Lemma and Latin transversals, Discrete Applied Mathematics 30 (1990), 151-154.

[4] A.M. Frieze and B. Pittel, Perfect matchings in random graphs with prescribed minimal degree, Trends in Mathematics, Birkhauser Verlag, Basel (2004), 95-132.

[5] G. Hahn and C. Thomassen, Path and cycle sub-Ramsey numbers and an edgecolouring conjecture, Discrete Mathematics 62 (1986), 29-33.

[6] W. Höeffding, Probability inequalities for sums of bounded random variables, Journal of the American Statistical Association 58 (1963), 13-30.

[7] R. Rue, Comment on [1]. 